\title{
Structural studies and influence of the structure on the electrical and optical properties of microcrystalline silicon thin films produced by RF sputtering
}

\author{
M. F. Cerqueira ${ }^{a}$, J.A. Ferreira ${ }^{\text {a }}$ G.J. Adriaenssens ${ }^{b}$ \\ ${ }^{a}$ Departamento de Física, Universidade do Minho, Campus de Gualtar, Braga, 4710-057 Portugal \\ ${ }^{b}$ Halfgeleiderfysica, Departement Natuurkunde, K.U. Leuven, Belgium
}

\begin{abstract}
Microcrystalline silicon thin films were produced by reactive magnetron sputtering on glass substrates under several different conditions (RF power and gas mixture composition). The film structure was studied by X-ray diffractometry (XRD), transmission electron microscopy (TEM) and Raman spectroscopy, allowing the determination of crystal sizes, crystallinity and mechanical strain. These parameters were evaluated by fitting a pseudo-Voigt function to the X-ray data, and by the application of the strong phonon confinement model to the Raman spectra. The degree of crystallinity and the presence of single crystals or crystal agglomerates, which was confirmed by TEM, depends on the preparation conditions, and strongly affects the optical spectra and the electrical transport properties.
\end{abstract}

Keywords: Microcrystalline silicon; Raman scattering; X-ray diffraction; Transmission electron microscopy; Absorption coefficient; Conductivity

\section{Introduction}

Solids made out of nano(micro)particles have physical properties different from those of the bulk material due to electronic or vibrational confinement effects, and also due to surface effects. Hydrogenated microcrystalline silicon ( $\mu \mathrm{c}-\mathrm{Si}: \mathrm{H})$ has attracted considerable attention, due to a higher electrical conductivity of this material compared to amorphous silicon $\left(\sigma_{\mu \mathrm{c}-\mathrm{Si}: \mathrm{H}}>\sigma_{\mathrm{a}-\mathrm{Si}: \mathrm{H}}\right)$, while preserving the inexpensive method of preparation. The electrical conductivity, and other physical properties relevant for many applications, are strongly influenced by the thin film microstructure of $\mu \mathrm{c}-$ $\mathrm{Si}: \mathrm{H}$. Nevertheless, from a theoretical point of view, a large number of questions remain unanswered [1].

Microcrystalline silicon thin films are generally described as consisting of small crystals (c-Si) embedded in an amorphous matrix (a-Si:H). The grain size ranges typically from 3 to $100 \mathrm{~nm}$, whereby the lower limit is defined by thermodynamic considerations [1]. 
This work will describe some experiments performed to obtain data concerning the structure of thin films produced by RF sputtering, and measurements of their electrical and optical properties. We will try to describe these properties as a function of the sample structure (overall crystallinity, c; and crystal size, D) and of the topological arrangement of the crystals in the matrix.

For the purpose of the structural characterization, we used X-ray diffractometry (XRD), transmission electron microscopy (TEM) and Raman spectroscopy. The latter is a very useful technique for studying the structure, geometry and type of nanoparticles. In the optical study, we used the transmission and reflection spectra in the visible range, and for the electrical properties, a conventional steadystate conductivity set-up was used. In this work, we also present the effects on the film properties of the crystal distribution and hydrogen fraction content present during the film growth. We will describe two kinds of transport mechanism according to the crystal distribution in the matrix.

\section{Experimental}

Microcrystalline silicon thin films were produced by reactive RF sputtering on Corning glass substrates as reported before [2]. Three different series were prepared: series $b$ and e were grown in an argon-rich atmosphere, and series $\mathrm{d}$ was grown in a hydrogen-rich atmosphere. The deposition conditions are summarized in Table 1 . The temperature was maintained constant at $\mathrm{T}=250^{\circ} \mathrm{C}$ through the film growth. Table 2 shows the growth conditions for some samples of each series. For the e series, only one sample was grown at an RF power lower than $300 \mathrm{~W}(\mathrm{e} 0)$. The thickness of the films varied from 0.24 to $2 \mu \mathrm{m}$. X-ray diffraction spectra were obtained with a Siemens X-ray Diffractometer D5000 using the $\mathrm{Cu} \mathrm{K} \alpha_{1}$ line. The sample was measured at grazing incidence (0.58).

Table 1

Growth conditions of the three different series

\begin{tabular}{llllll}
\hline & RF power $(\mathrm{W})$ & $p_{\mathrm{H}_{2}}(\mathrm{~Pa})$ & $p_{\mathrm{Ar}}(\mathrm{Pa})$ & $p_{\mathrm{H}_{2}} /\left(p_{\mathrm{H}_{2}}+p_{\mathrm{Ar}}\right)$ & Deposition rate $(\mathrm{nm} / \mathrm{s})$ \\
\hline Series $b$ & $40-300$ & 0.15 & 0.78 & 0.16 & $0.04-0.33$ \\
Series $e$ & 300 and 400 (one sample at 200) & 0.15 & 0.78 & 0.16 & $0.41-0.62(0.28)$ \\
Series $d$ & 80 and 150 & 0.35 & 0.23 & 0.6 & $0.02-0.03$ \\
\hline
\end{tabular}

Raman scattering measurements were performed in a Jobin Ivon MicroRaman spectrometer with a triple monochromator using the $514.5 \mathrm{~nm}$ line of an argon laser. A liquid nitrogen-cooled charge coupled device (CCD) was used to detect the scattered light. The laser power was kept low enough to avoid modifying the amorphous to crystalline ratio of the samples. 
Samples for TEM observations were prepared by standard procedures, including mechanical and ionmill techniques. The TEM observations were made using a Hitachi H-9000 electron microscope operated at $300 \mathrm{kV}$.

For the conductivity measurements, thermally evaporated coplanar Al contacts were used. The dark conductivity, $\sigma_{\mathrm{d}}$, was measured between 200 and $400 \mathrm{~K}$, using a constant applied voltage (10 V) in vacuum conditions.

The absorption coefficient, $\alpha$, was obtained from the transmission and reflection spectra measured by a Shimadzu double beam spectrometer in the range of 200-3200 nm.

\begin{tabular}{|c|c|c|c|c|}
\hline Sample & RF power (W) & $p_{\mathrm{H}_{2}}(\mathrm{~Pa})$ & $p_{N}(\mathrm{~Pa})$ & Thickness, $d(\mathrm{~nm})$ \\
\hline$d \theta$ & 80 & 0.35 & 0.23 & 241 \\
\hline d10 & 150 & 0.35 & 0.23 & 1760 \\
\hline$e 0$ & 200 & 0.15 & 0.78 & 1362 \\
\hline el6 & 300 & 0.15 & 0.78 & 637 \\
\hline b5 & 100 & 0.15 & 0.78 & 632 \\
\hline
\end{tabular}

\section{Structure}

\subsection{X-ray diffraction}

Fig. 1a shows the X-ray spectra of some representative $\mu \mathrm{c}-\mathrm{Si}: \mathrm{H}$ thin films, where the three diffraction peaks of c-Si are seen. The relatively large background under the (111) diffraction peak is attributed to the amorphous silicon matrix, with some contribution from the glass substrate. The $\mathrm{e}_{0}$ sample (grown at a lower RF power) is the only one of the e series showing X-ray peaks (microcrystalline).

All the other e samples are amorphous.

By fitting a pseudo-Voigt function $(\mathrm{pV}(\mathrm{x}))$ to each diffraction peak [3], it is possible to obtain the average crystal size (D) and the thin film strain (1 ) of the crystals.

The pseudo-Voigt function is a sum of $\mathrm{n}$ Cauchy functions (C) with $\mathrm{m}$ Gauss functions $(\mathrm{G})$, given by Eq. (1)

$$
p V(x)=I_{\mathrm{p}}(\eta C(x)+(1+\eta) G(x))
$$

where Ip is the maximum intensity of the diffraction peak, and $\eta$ is the fraction of Cauchy component present in the peak. 
According to this model, the average crystal size (D) and the thin film strain ( $\varepsilon$ ) are given by Eqs. (2) and (3) [3]

$$
\begin{aligned}
& D=\frac{\lambda}{\beta_{\mathrm{C}} \cos \theta} \\
& \left\langle\varepsilon^{2}\right\rangle^{0.5}=\frac{\beta_{\mathrm{G}}}{4 \tan \theta}
\end{aligned}
$$

where $\beta_{\mathrm{C}}$ and $\beta_{\mathrm{G}}$ are the integral breadths of the Cauchy and Gaussian components, respectively. $\lambda$ is the wavelength of the copper $\mathrm{K} \alpha_{1}$ radiation $(\lambda=1.50456 \mathrm{~A})$, and $\theta$ is the diffraction angle.
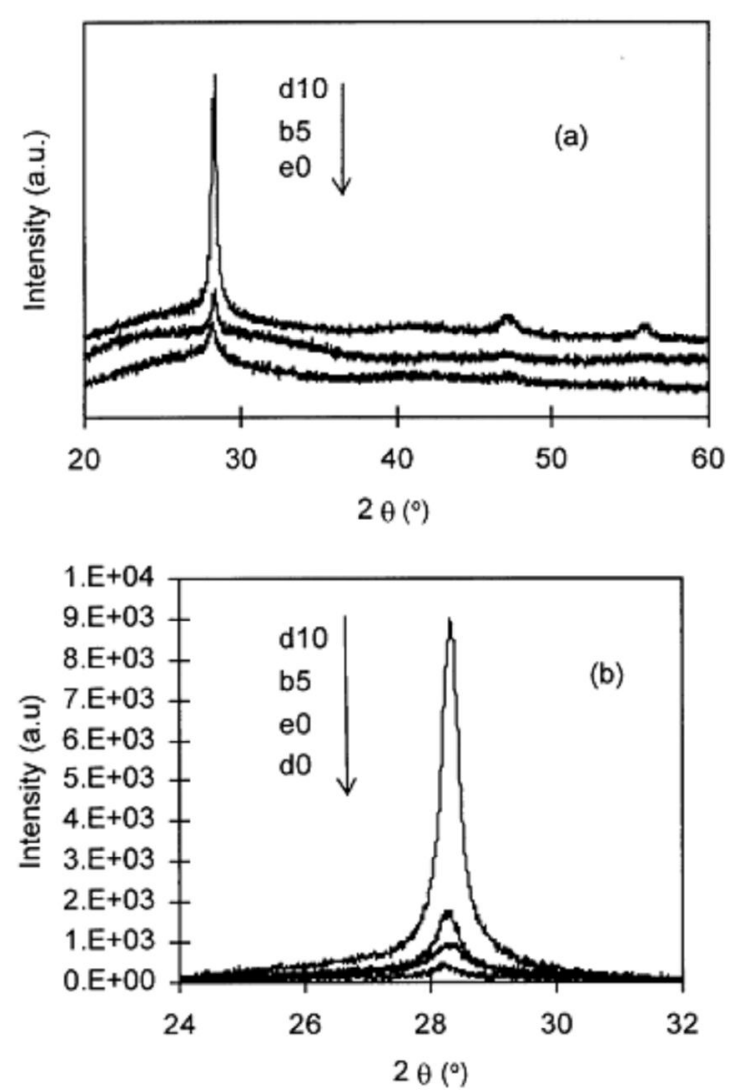

Fig. 1. (a) X-ray spectra of some microcrystalline silicon samples. (b) Detail of (111) peak of each sample.

Corrections for background, spectral width, $\mathrm{K} \alpha_{2}$ radiation and instrumental peak broadening were performed following methods described elsewhere [4]. The analyzed peaks are shown in Fig. 1b. From the $\mathrm{X}$-ray line profiles, the values for the average grain sizes $\left(\mathrm{D}_{\mathrm{X}}\right)$ and strain $\left(\varepsilon_{\mathrm{X}}\right)$ obtained are presented in Table 3. 
Table 3

Crystalline fraction, average values of grain size and strain obtained from the TEM pictures and determined by the fittings of the Raman and X-ray peaks ${ }^{2}$

\begin{tabular}{llllccc}
\hline Sample & $D_{\mathrm{X}} \pm 25(\AA)$ & $\varepsilon_{\mathrm{X}}(\%)$ & $D_{\mathrm{R}} \pm 10(\AA)$ & $D_{\mathrm{T}} \pm 10(\AA)$ & $c_{\mathrm{R}}(\%)$ & Crystal distribution \\
\hline$d 0$ & 199 & 0.9 & 75 & $>60$ & 50 & Agglomerated \\
$e 0$ & 104 & 1.3 & 55 & 70 & 45 & Isolated \\
d10 & 163 & 0.8 & 55 & 50 & Agglomerated & 55 \\
b5 & 156 & 1.5 & lsolated & 55 \\
\hline
\end{tabular}

${ }^{a} D$, average values of grain size; $\varepsilon$, strain; $c$, crystalline fraction. Subscripts $\mathrm{R}, \mathrm{T}$ and $\mathrm{X}$ indicate Raman, TEM and X-ray, respectively. The uncertainties relative to each measurement technique are indicated in parentheses.

\subsection{Raman spectroscopy}

The Raman spectra of some of our samples are shown in Fig. 2a.
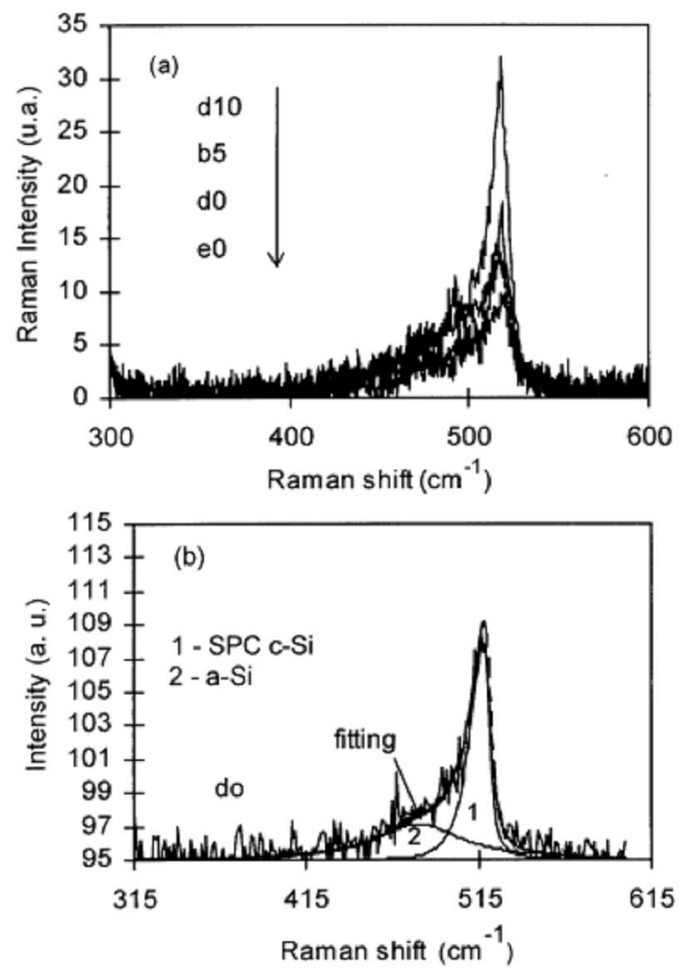

Fig. 2. (a) Raman spectra of some $\mu$-Si:H samples. (b) A typical Raman fitting (sample $d O$ ).

To analyze the Raman spectra, we used a Gaussian profile for the amorphous structure (transverse optical mode; TO), and the crystalline profile is calculated based on the strong phonon confinement model $[5,6]$ according to Eq. (4)

$$
I(\omega)=\int_{0}^{\infty} \frac{\exp \left(-q^{2} D^{2} / 16 \pi^{2}\right) \mathrm{d}^{3} q}{(\omega-\omega(q))^{2}+(\Gamma / 2)^{2}}
$$


where $\omega(\mathrm{q})$ is the phonon dispersion curve, $\mathrm{q}$ is expressed in units of $2 \pi / a$, with $a$ as the lattice constant, and $\omega$ is the phonon energy at $\mathrm{q}=0\left(\omega=521 \mathrm{~cm}^{-1}\right)$. $\mathrm{D}$ is the crystal size, and $\Gamma$ is the natural line-width of crystalline silicon.

This equation assumes that the shape of the crystals is spherical. In order to simplify the calculations, we used a spherical Brillouin zone (BZ) and considered the phonon dispersion curve as isotropic. Fig. $2 \mathrm{~b}$ shows a typical fitting to the Raman curves. The shape of the crystals was confirmed by the TEM images, and the other two assumptions are justified because only a small region near the centre of the $\mathrm{BZ}$ contributes to the scattering.

By applying the strong phonon confinement model, we obtain the crystal size $\left(D_{R}\right)$, and it is possible to calculate the crystalline fraction $\left(c_{R}\right)$ using the integrated intensities of the amorphous and crystalline components [2,7]. Our results are presented in Table 3.

\subsection{TEM}

From the TEM analysis, information concerning the crystal distribution, average crystal size, and the presence or absence of strain was obtained. Fig. 3 shows the diffraction images of some samples; two of the $\mathrm{d}$ series, one of the $\mathrm{b}$ series and one of the e series. The rings (due to the planes of $\mathrm{c}-\mathrm{Si}$ ) are clearer in the $\mathrm{d} 0$ and d10 samples (grown in a hydrogen-rich atmosphere) than in b5, illustrating that samples from the $\mathrm{d}$ series are more crystalline. The plane spacing, obtained using a horizontal profile through the centre of the diffraction images, is in agreement with literature data for $\mathrm{c}-\mathrm{Si}$, indicating that the crystals are almost stress free.

Fig. 4 shows a surface photograph of the d10 and b5 samples. We verify from these dark field images with the same magnification, that the crystals in the sample grown in an argon-rich atmosphere (b5) are dispersed on the matrix, while on the other sample they form agglomerates of several crystals. This dependence of crystal distribution on the kind of atmosphere used was verified for several samples. 

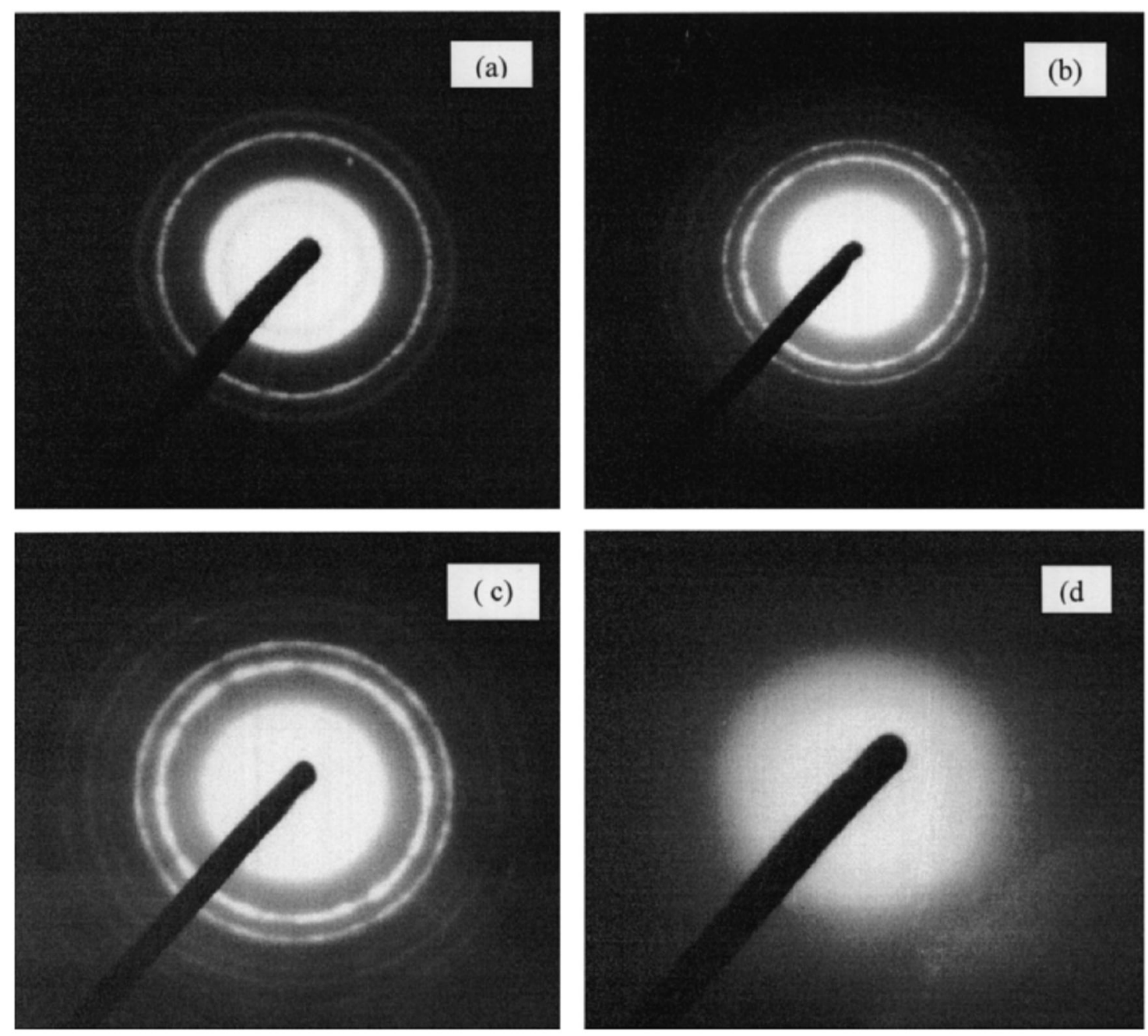

Fig. 3. TEM difractograms of microcrystalline samples and of an amorphous sample: (a), b5; (b), d0; (c), d10; and (d), el6.

The cross-sectional views depicted in Fig. 5 show that the crystals have a compact, rather than elongated shape (approximately spherical). The two images are of the same region of the sample, but originating from different relative orientations between the electron beam and sample.

Table 3 summarizes the crystal size obtained by imaging analysis $\left(\mathrm{D}_{\mathrm{T}}\right)$, with information on the type of crystal distribution on the matrix. 

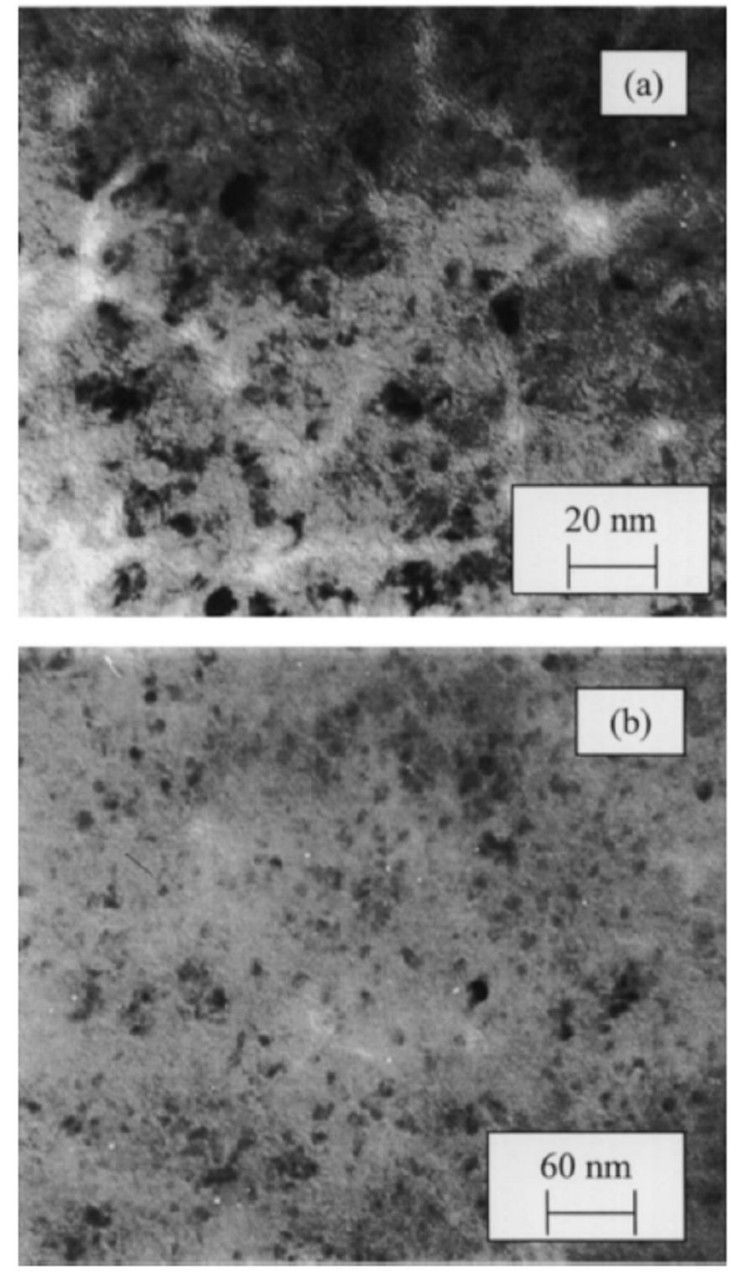

Fig. 4. Surface dark field TEM photographs ( $300 \mathrm{~K}$ magnification) of some $\mu \mathrm{c}$-Si:H samples: (a), $d 10$; and (b), b5.
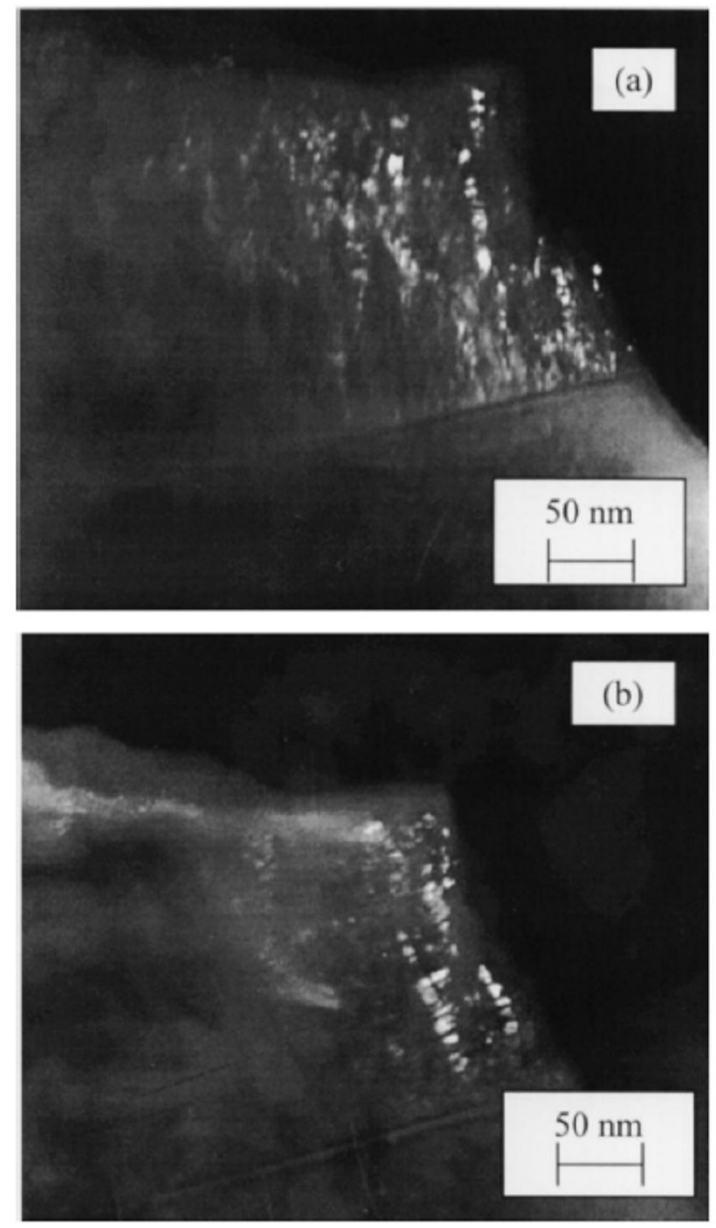

Fig. 5. Dark field cross-sectional TEM photograph for the $b 5$ sample ( $\mu \mathrm{c}$ $\mathrm{Si}: \mathrm{H}$ ), with $200 \mathrm{~K}$ magnification; (a) and (b) are of the same sample region showing different crystals (with different orientations).

\section{Structural discussion}

From Table 3, we observe that the crystal sizes as determined from the XRD experiments are larger than the ones determined by Raman spectroscopy and TEM. There are two reasons for this difference. The first is based on the fact that Raman scattering yields information about the smallest crystalline dimension, while XRD results refer to an average value for the three directions. On the other hand, Xray data are related to the coherence length, which must include more than one crystal, according to the TEM crosssectional pictures. 
From Table 3, we also verify that the crystal strain (from XRD analysis) is lower in the samples having agglomerates of crystals compared with the samples having isolated crystals, in agreement with theoretical predictions.

Table 4 presents the structural analysis summary for the three different series as a result of the different techniques used. For the crystal size, we present Raman values, since they are in good agreement with the TEM values.

Table 4

Structural parameters of the samples

\begin{tabular}{lccll}
\hline & $c(\%)$ & $D(\AA)$ & Crystal distribution & Atmosphere \\
\hline Series $b$ & $45-60$ & $55-60$ & Isolated & Ar-rich \\
Series $e$ (except $e 0$ sample) & 0 & 0 & - & Ar-rich, but high deposition rates \\
Series $d$ & $60-65$ & $60-66$ & Agglomerates & $\mathrm{H}_{2}$-rich \\
\hline
\end{tabular}

\section{Electrical and optical properties}

\subsection{Electrical conductivity}

The dependence of the room temperature dark conductivity $\left(\sigma_{\mathrm{d}}\right)$ on deposition rate $(\mathrm{rd})$ is shown in Fig. 6. For deposition rates lower than $0.1 \mathrm{~nm} / \mathrm{s}$, we observe an increase of $\sigma_{\mathrm{d}}$ with deposition rate. For deposition rates higher than $0.1 \mathrm{~nm} / \mathrm{s}$, the conductivity decreases, since at higher depositions rates (rd), the sputtered particles have insufficient time to diffuse in the grown film in order to find the more favourable arrangement (crystalline), and the material produced is largely amorphous.

Fig. 7 shows the behaviour of the room temperature dark conductivity $\left(\sigma_{d}\right)$ with sample crystallinity (c). The dark conductivity increases with the crystallinity, since the electrical conductivity of the crystalline fraction is higher than the electrical conductivity of the amorphous component $\left(\sigma_{\mathrm{d}}(\mathrm{c}-\mathrm{Si})>\right.$ $\left.\sigma_{\mathrm{d}}(\mathrm{a}-\mathrm{Si})\right)$.

The temperature dependence of the conductivity $\left(\sigma_{d}\right)$ can be approximated for all the samples by a simple, thermally activated process. However, more detailed study does reveal that the transport properties depend on the electronic band structure of the three different components in the material; the crystalline, amorphous and mixed phase which may be found in the grain boundaries.

Nevertheless, applying the thermally activated conductivity relation (Eq. (5))

$$
\sigma_{\mathrm{d}}=\sigma_{0} \exp \left(-E_{\mathrm{a}} / k T\right)
$$

to the data, we can extract the activation energy $\left(\mathrm{E}_{\mathrm{a}}\right)$ and the pre-factor of the conductivity $\left(\sigma_{\mathrm{o}}\right)$. Our results indicate that the $\sigma_{\mathrm{o}}$ value varies between $10^{-5}$ and $10^{2}(\Omega \mathrm{cm})^{-1}$ for the amorphous samples, and 
between $10^{-1}$ and $10^{4}(\Omega \mathrm{cm})^{-1}$ for $\mu \mathrm{c}-\mathrm{Si}: H$ samples. Fig. $8 \mathrm{a}$, b shows the $\sigma_{\mathrm{o}}$ dependence on the $\mathrm{E}_{\mathrm{a}}$. The solid line is the theoretical fitting of the Meyer-Neldel rule [8,9] according to Eq. (6)

$$
\sigma_{0}=A \exp \left(\frac{E_{\mathrm{a}}}{E_{0}}\right)
$$

where $\mathrm{A}$ is a positive constant, and $\mathrm{E}_{0}$ is the characteristic energy of the process. Even though not wellunderstood, this relationship has been consistently observed in disordered semiconductors.

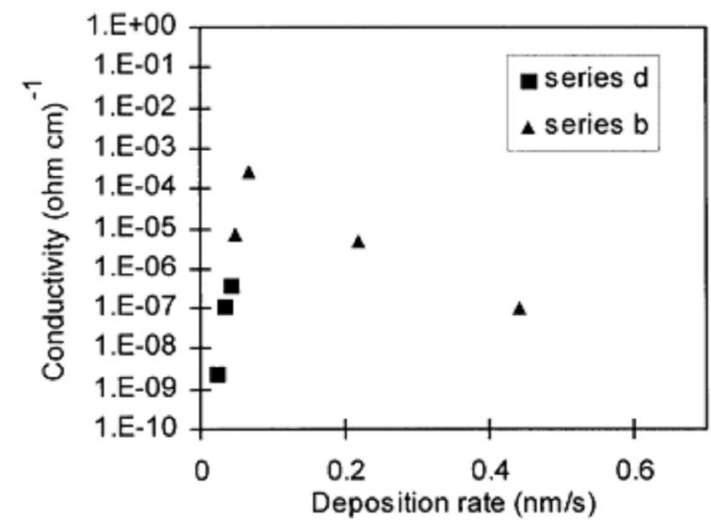

Fig. 6. Behaviour of the room temperature dark conductivity with deposition rate.

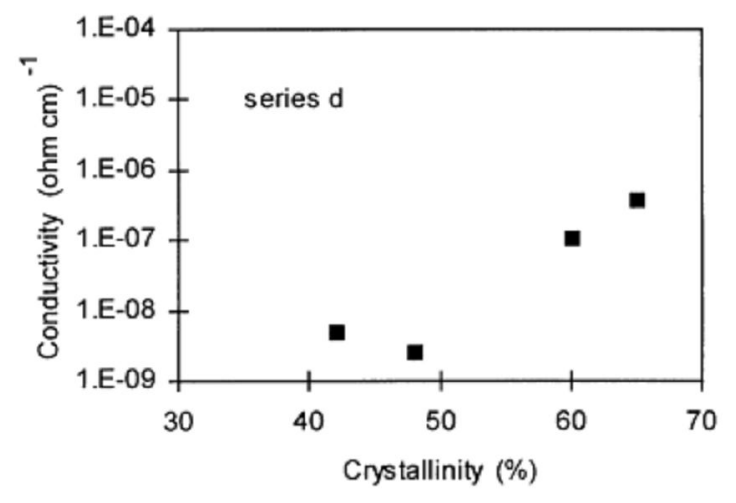

Fig. 7. Behaviour of the room temperature dark conductivity with crystallinity of the samples.

The Meyer-Neldel parameters obtained for the $\mathrm{d}$ and e series are different and in agreement with the values obtained by other workers. For the $\mathrm{d}$ series $\left(\mathrm{A}=1 \times 10^{22}(\Omega \mathrm{cm})^{-1} ; \mathrm{E}_{0}=0.06 \mathrm{eV}\right)$, they agree with the usual values reported for $\mu \mathrm{c}-\mathrm{Si}: \mathrm{H}$ samples $\left(\mathrm{E}_{0}=0.08 \mathrm{eV}\right)$, and for the e series $\left(\mathrm{A}=1 \times 10^{-6}(\Omega\right.$ $\mathrm{cm}^{-1}, \mathrm{E}_{0}=0.028 \mathrm{eV}$ ), they are in agreement with those obtained using Monte Carlo simulations for aSi:H $\left(\mathrm{E}_{0}=0.035 \mathrm{eV}\right)[10]$.

In order to obtain better fittings and more information on the transport mechanism, we have applied several transport models to our $\sigma_{\mathrm{d}}$ vs. T curves, namely the grain boundary theory model (GBT) [11,12], the heteroquantum dots model (HQD) [13-15], and the two-phase model of Demichelis et al [16]. In all of these models, the potential barriers that exist in this kind of mixed system play an important role. Electrons can cross the barriers by two different mechanisms: the thermoionic emission and the tunnel effect. Although a more or less satisfactory agreement with the experimental data can be obtained with each of the models, the best overall picture emerges with the Demichelis model. 

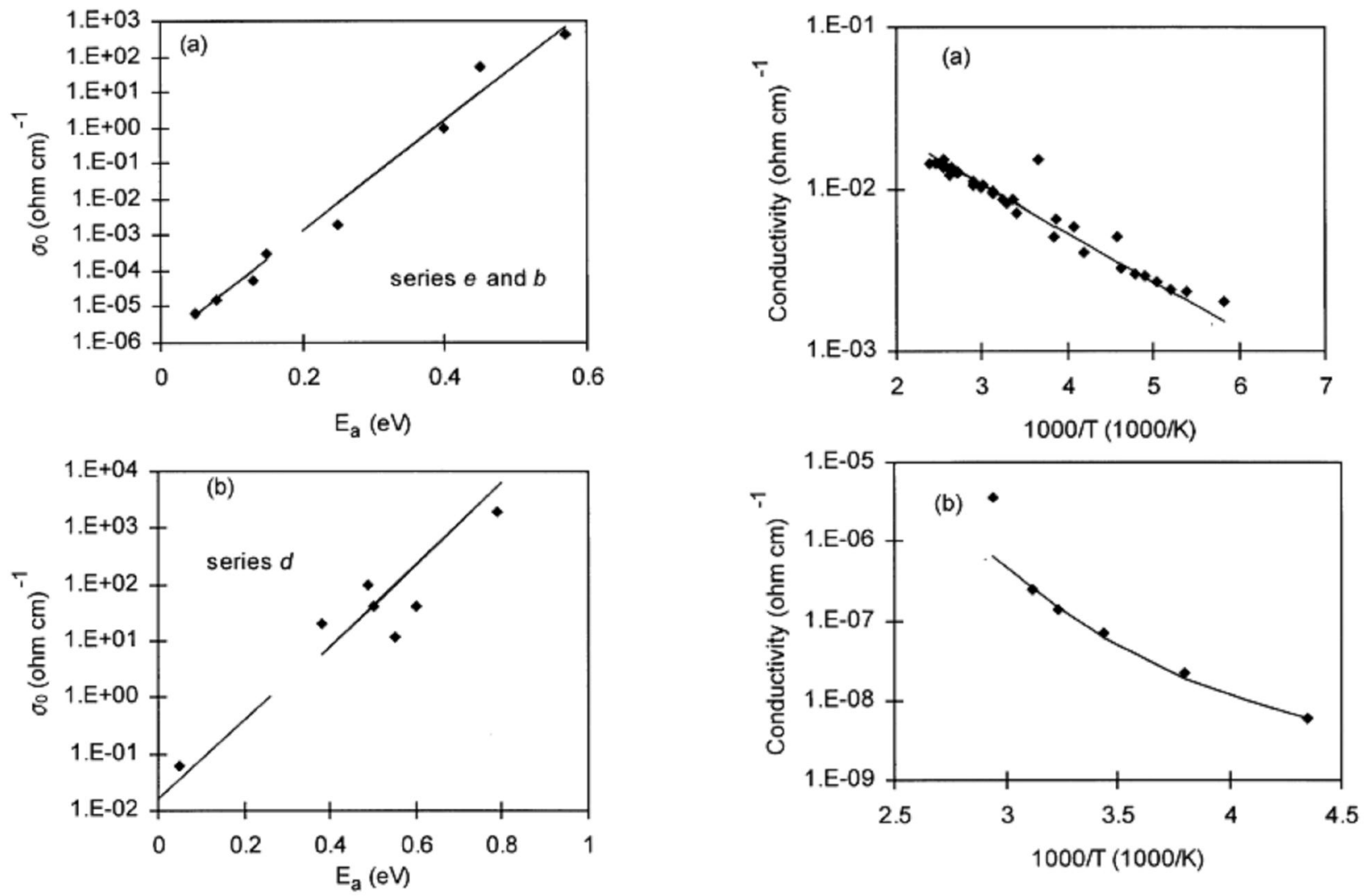

Fig. 8. $\sigma_{0}$ dependence on the $E_{2}$ for: (a), the $e$ and $b$ series; (b), the $d$ series.

Fig. 9. Conductivity fittings according to the Demichelis model for: (a), a sample having agglomerate crystals; and (b), a sample having isolated crystals. Full lines are theoretical curves for: (a), thermoionic emission; and (b), tunnelling.

According to this model, the electrical transport can occur by thermoionic emission from the c-Si to the $\mathrm{CB}$ of the a-Si component, or by a tunnel effect (hopping) from a silicon crystal to another crossing the amorphous matrix. The probability $(\mathrm{P})$ of tunnelling is a function of the mean distance between microcrystals $(\mathrm{L})$, the decay rate of the electronic wavefunction of a localized carrier on a single atomic well (a), and the attempt-to-escape frequency $v_{0}$, according to Eq. (7) [16]

$$
P=2 \mathrm{e} L^{2} \exp (-2 L / a) \nu_{0}
$$

Our results indicate that the transport mechanism is dependent on the crystal distribution on the matrix. In the case of isolated crystals, the transport mechanism is the tunnel effect, and in the case of agglomerates of crystals, the transport is dominated by thermoionic emission, as shown in Fig. 9a,b, respectively. Table 5 presents the parameters obtained for our samples using the Demichelis model, 
together with the results obtained by Demichelis [16] on the basis of the Komuro $\mu \mathrm{c}-\mathrm{Si}: \mathrm{H}$ data [17]. During the fitting process, some parameters, such as $v_{0}, a, \mu \mathrm{c}$ and $\mu \mathrm{a}$, have been fixed. We have verified that small variations $( \pm 0.1 \mathrm{eV})$ on $\mu \mathrm{c}$ and $\mu \mathrm{a}$ (compared to Demichelis values [16]) yield worse fittings.

\begin{tabular}{|c|c|c|c|c|}
\hline & \multicolumn{4}{|c|}{$\mu c-S i: H$ samples } \\
\hline & Series $b$ & $e 0$ & Series $d$ & $\mu c-S i: H[16]$ \\
\hline$N_{0}\left(\mathrm{~cm}^{-3}\right)$ & $1 \times 10^{22}$ & $1 \times 10^{19}$ & $1 \times 10^{20}$ & $1 \times 10^{20}$ \\
\hline$\mu_{c}\left(\mathrm{~cm}^{-2} \mathrm{~s}^{-1} \mathrm{~V}^{-1}\right)$ & - & - & 3 & 3 \\
\hline$\mu_{a}\left(\mathrm{~cm}^{-2} \mathrm{~s}^{-1} \mathrm{~V}^{-1}\right)$ & 1 & 1 & & 1 \\
\hline$E_{\mathrm{a}}(\mathrm{eV})$ & $0.15-0.3$ & 0.1 & - & 0.15 \\
\hline$E_{\mathrm{a}}^{\prime}(\mathrm{eV})$ & - & - & $0.4-0.5$ & 0.2 \\
\hline$E^{\prime \prime}{ }_{a}(\mathrm{eV})$ & $0.3-0.55$ & 0.45 & - & 0.4 \\
\hline$\nu_{0}\left(\mathrm{~s}^{-1}\right)$ & $10^{12}$ & $10^{12}$ & - & $10^{12}$ \\
\hline$a(\AA)$ & 15 & 15 & - & 15 \\
\hline$L(\AA)$ & $350-380$ & 365 & - & 300 \\
\hline
\end{tabular}

The uncertainties on $E_{a}, \mathrm{E}_{a}^{\prime}$ and $\mathrm{E}_{a}^{\prime \prime}$ values are less than $0.02 \mathrm{eV}$.

In Table $5, \mathrm{E}_{\mathrm{a}}$ is $\mathrm{E}_{\mathrm{C}}(\mathrm{c}-\mathrm{Si})-\mathrm{E}_{\mathrm{F}}$, and $\mathrm{E}_{0}^{\prime \prime}$ is $\mathrm{E}_{\mathrm{a}}+\mathrm{E}_{\mathrm{b}}$, where $\mathrm{E}_{\mathrm{b}}$ is the energy difference between the conduction band minimum of a-Si:H (matrix) and c-Si (crystal). $\mathrm{N}_{0}$ is the density of localized states. From Table 5, we verify that our results are in good agreement with the results obtained by Demichelis.

\subsection{Absorption coefficient}

In single crystals, the Tauc's plot of $(\alpha \mathrm{E})^{1 / 2}$ vs. E gives a well defined optical gap. In amorphous materials, the definition of an optical gap is less straightforward, since the transition from the transparent to the absorbing region is gradual. Microcrystalline material does show an intermediate behaviour, with the absorption curves strongly dependent on the deposition conditions, as shown in Fig. 10a. The different symbols refer to samples grown with different RF power, according to Table 1 . Using the Tauc's plot, the value of the energy gap for these microcrystalline samples is higher than 2 $\mathrm{eV}$. 

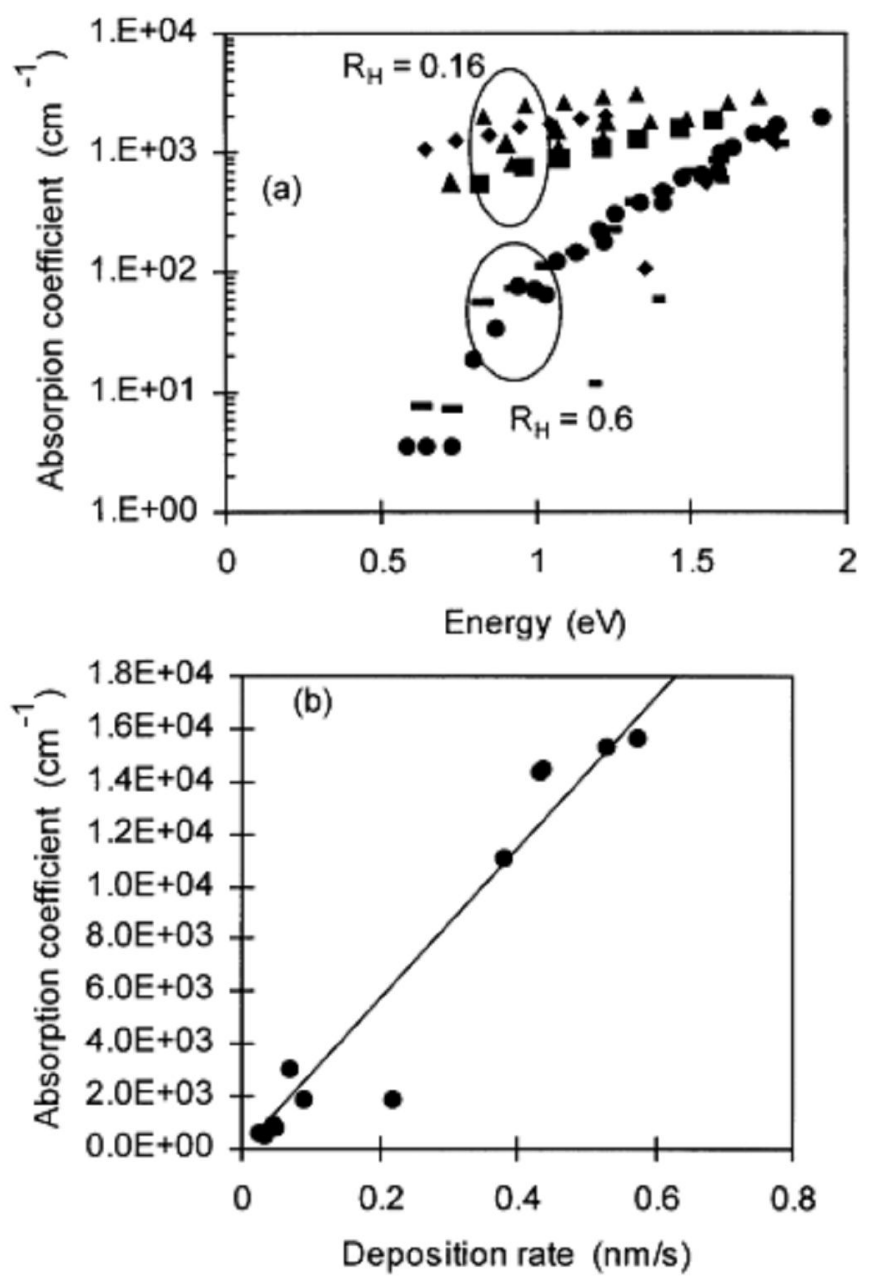

Fig. 10. (a) Behaviour of the absorption coefficient with energy and hydrogen fraction $\left(R_{\mathrm{H}}\right)$. (b) Behaviour of the absorption coefficient at $1.6 \mathrm{eV}$ with deposition rate.

Since, for energies below the optical gap, the absorption involves subgap states, a lower absorption coefficient shows a decrease in dangling bond density. Comparing the absorption coefficients for those energies thus allows us to conclude the relative abundance of defect states.

Fig. 10b shows the absorption coefficient (at $1.6 \mathrm{eV}$ ) as a function of deposition rate. It is visible that the absorption coefficient of the amorphous samples (higher RF power) is much higher than the values for the microcrystalline samples. As the optical quality of the sample is related to the transparency of the sample for low energies, the results shows that the presence of microcrystals improves the optical quality.

For energies below the optical gap energy, we verify, from Fig. 10a, that a high fraction of hydrogen 
$\left(\mathrm{R}_{\mathrm{H}}=\mathrm{pH}_{2} /\left(\mathrm{pH}_{2}+\mathrm{pAr}\right)\right)$ during the deposition favours the growth of microcrystals, the elimination of some of the weak $\mathrm{Si}-\mathrm{Si}$ bonds which are present in the amorphous phase, and the further hydrogen passivation of the dangling bonds.

The absorption coefficient value decreases with the sample crystallinity approaching the value for fully crystalline material (Fig. 11). We think that the lattice adjustments needed at the grain boundaries incorporate much of the strain, which, in pure a-Si:H, leads to the creation of dangling bond defects. This means that with crystal formation, the dangling bond density of the remaining amorphous component decreases and the optical quality improves.

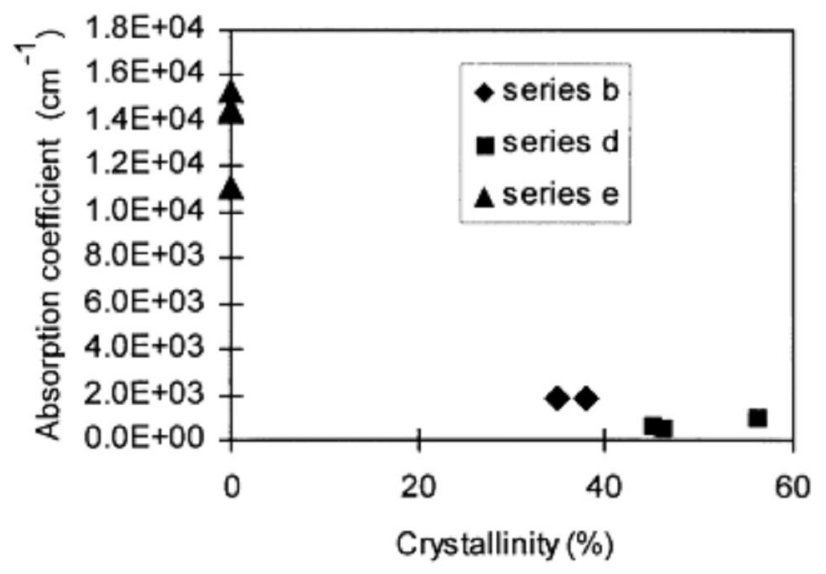

Fig. 11. Behaviour of the absorption coefficient at $1.6 \mathrm{eV}$ with crystalline fraction.

\section{Conclusions}

\subsection{Structure}

The microstructure of thin $\mu \mathrm{c}-\mathrm{Si}: \mathrm{H}$ films deposited by reactive magnetron sputtering on glass has been analyzed by TEM, XRD and Raman spectroscopy. These films consist of small Si crystals embedded in a matrix of amorphous Si. Crystal sizes, as determined by TEM measurements and Raman spectroscopy, were generally in good agreement. The crystal distribution in the matrix is strongly dependent on the type of atmosphere used during the growth process. The TEM analysis indicates that the crystals form agglomerates if the samples are grown in a hydrogen-rich atmosphere, otherwise the crystals are evenly dispersed in the matrix. 


\subsection{Optical properties}

By increasing the hydrogen fraction in the plasma $\left(\mathrm{R}_{\mathrm{H}}=\mathrm{pH}_{2} /\left(\mathrm{pH}_{2}+\mathrm{pAr}\right)\right)$, the density of the gap defects in the a-Si:H matrix decreases. This means that the absorption is lower in samples having agglomerates of crystals than in samples having isolated crystals.

Lower deposition rates produce more ordered material (higher optical gaps from the Tauc's plot). The optical quality improves by decreasing the RF power (decreasing deposition rate), since the time available for diffusion of the sputtered atoms on the surface of the growing film increases.

\subsection{Electrical properties}

The conductivity increases with the crystallinity. This behaviour supports the idea that, in this kind of mixed material, the electrical behaviour is essentially due to the crystalline component.

Our results give us the information that the transport mechanism is strongly dependent on the crystal distribution of the amorphous matrix. For agglomerates of crystals, the transport is dominated by thermoionic emission, and when isolated crystals are dispersed in the matrix, the tunnel effect is the controlling transport mechanism.

\section{Acknowledgements}

M.F. Cerqueira acknowledges a grant from PRAXIS XXI.

\section{References}

[1] G.J. Adriaenssens, J. Jansen, M.F. Cerqueira, Electronic, Optoelectronic and Magnetic Thin Films, in: J.M. Marshall, N. Kirov, A. Vavrek (Eds.), Proceedings of the Eight International School on Condensed Matter Physics 1994, Varna, Bulgaria, September 18- 23 (1995) p. 235.

[2] M.F. Cerqueira, M. Andritschky, L. Rebouta, J.A. Ferreira, M.F. Da Silva, Vacuum 46 (1995) 1385.

[3] H. Th, J.I. de Keijser, E.F. Langford, A.B.P. Mittemeyer, Rogels, J. Appl. Crystallogr. 11 (1978) 10.

[4] H.P. Klug, L.E. Alexander, X-ray Diffraction Procedures for Polycrystalline and Amorphous Materials, Wiley, New York (1980).

[5] I.H. Campbell, P.M. Fauchet, Solid State Commun. 58 (1986) 739.

[6] M. Yang, D. Huang, P. Hao, F. Zhang, X. Hou, X. Wang, J. Appl. Phys. 75 (1994) 651.

[7] M.F. Cerqueira, J.A. Ferreira, M. Andritschky, M.F.M. Costa, Microelectron. Eng. $43 / 44$ (1998) 627. 
[8] W. Beyer, H. Overhof, in: J.I. Pankove (Ed.), Hydrogenated Amorphous Silicon, Vol. 21C, Academic Press, New York (1984) p. 257.

[9] W. Meyer, H. Neldel, Z. Tech. Phys. 12 (1937) 588.

[10] W.C. Chen, L. Hamel, A. Yelon, J. Non-Cryst. Solids 220 (1997) 254.

[11] P.G. Lecomber, G. Willeke, W.E. Spear, J. Non-Cryst. Solids 59/60 (1983) 795.

[12] M. Komuna, H. Curtis, F.A. Sarott, S. Veprek, Philos. Mag. B 55 (1987) 377.

[13] G.Y. Hu, R.F. O'Connell, Y.L. He, M.B. Yu, J. Appl. Phys. 78 (1995) 3945.

[14] M. Cuniot, Y. Marfaing, J. Non-Cryst. Solids 78/79 (1985) 978.

[15] F. Evangelisti, J. Non-Cryst. Solids 78/79 (1985) 969.

[16] F. Demichelis, C.F. Pirri, E. Tresso, Philos. Mag. B 67 (1993) 331.

[17] S. Komuro, Y. Aoyagi, Y. Segawa, et al., J. Appl. Phys. 56 (1984) 1658. 